\title{
Neuropsychological Correlates of MRI Measures in the Continuum of Cognitive Decline at Old Age
}

\author{
Wiesje M. van der Flier ${ }^{a}$ Huub A.M. Middelkoop ${ }^{b}$ \\ Annelies W.E. Weverling-Rijnsburger ${ }^{c}$ Faiza Admiraal-Behloul ${ }^{d}$ \\ Eduard L.E.M. Bollen ${ }^{\mathrm{b}}$ Rudi G.J. Westendorp ${ }^{\mathrm{c}}$ Mark A. van Buchem ${ }^{\mathrm{e}}$ \\ ${ }^{a}$ Department of Neurology and Alzheimer Center, VU Medical Center, ${ }^{b}$ Department of Neurology, \\ ${ }^{\mathrm{c}}$ Departments of Gerontology and Geriatrics, ${ }^{\mathrm{d}}$ Section of Image processing (LKEB) of the Department of \\ Radiology and ${ }^{\mathrm{e}}$ Department of Radiology, Leiden University Medical Center, Leiden, The Netherlands
}

\section{Key Words}

MRI $\cdot$ White matter hyperintensities $\cdot$ Medial

temporal lobe $\cdot$ Elderly

\begin{abstract}
Objective: To investigate the independent associations between medial temporal lobe atrophy and white matter hyperintensities (WMH) and cognitive functions in the elderly. Methods: Cognitive functions of 41 Alzheimer's disease patients, 20 patients with mild cognitive impairment and 28 elderly subjects without memory complaints were assessed using a neuropsychological test battery. Quantitative MRI measures of medial temporal lobe volume and WMH were obtained. Multiple regression analyses were performed to assess the independent contribution of MRI measures to impairment in several cognitive functions. Results: Scores on the Wechsler Memory Scale and Trails B depended selectively on medial temporal lobe volume, whereas WMH selectively contributed to performance on Trails A. Medial temporal lobe volume and $\mathrm{WMH}$ both contributed to scores on the Cambridge Cognitive Examination and the Boston nam-
\end{abstract}

ing task. Conclusions: MRI measures suggestive of Alzheimer-type pathology and microvascular pathology independently contribute to cognitive decline at old age. Memory impairment as measured using the Wechsler Memory Scale and performance on Trails B primarily depended on medial temporal lobe atrophy. Psychomotor slowness, as measured using Trails A, mainly depended on $\mathrm{WMH}$. These results suggest that vascular pathology and Alzheimer-type pathology each have specific cognitive correlates.

Copyright $\odot 2005$ S. Karger AG, Basel

\section{Introduction}

Alzheimer's disease (AD) is a neurodegenerative disorder characterized by gradually increasing cognitive impairment [1]. The clinical syndrome of AD does not develop abruptly. Rather, there is a continuum of cognitive decline at old age with cognitively healthy elderly at one end of the spectrum, and patients with AD at the other end. The syndrome of mild cognitive impairment (MCI) resides within this continuum of cognitive decline $[2,3]$.

\section{KARGER \\ Fax +4161306 1234 E-Mail karger@karger.ch} www.karger.com
(C) 2005 S. Karger AG, Basel $1420-8008 / 05 / 0203-0082 \$ 22.00 / 0$

Accessible online at: www.karger.com/dem
W.M. van der Flier

Department of Neurology and Alzheimer Center, VU Medical Center PO Box 7057

NL-1007 MB Amsterdam (The Netherlands)

Tel. +31 20444 1079, Fax +31 20444 0715, E-mail wm.vdflier@vumc.nl 
In analogy, the neuropathological changes presumed to underly the cognitive decline in AD and MCI also develop gradually. From neuropathological studies, it is known that Alzheimer-type (i.e., neurofibrillary tangles and senile plaques) and vascular pathological changes are the most important correlates of cognitive impairment, with no clear thresholds predicting dementia status $[4,5]$. Both types of abnormalities are frequently observed in subjects with dementia as well as in cognitively normal elderly, confirming the notion of a continuum of both cumulative neuropathology and cognitive decline [4]. The clinical syndrome of dementia may occur when the combined burden of pathology reaches a certain threshold [6].

On MRI, medial temporal lobe atrophy presumably reflects Alzheimer-type pathology, whereas white matter hyperintensities (WMH) are of vascular origin $[7,8]$. Medial temporal lobe atrophy and WMH have been shown to coexist in $\mathrm{AD}[9,10]$. There have been a number of studies addressing the associations between single MRI measures and impairment in cognitive domains. Medial temporal lobe atrophy has been shown to be associated with cognitive decline in AD and MCI [11-15]. The medial temporal lobes are crucial in memory, which is usually the first cognitive function to deteriorate in $\mathrm{AD}$ [16]. WMH have been reported to be associated with poor performance on tasks involving speed in healthy elderly [17, 18]. $\mathrm{WMH}$ are more common in patients with AD than in healthy elderly, and associations with dementia severity have been found, although not consistently [19-22]. When evaluated in mono-factorial studies, both medial temporal lobe atrophy and WMH seem to be associated with cognitive decline. However, as yet it has not been clarified if these measures jointly contribute to the severity of cognitive impairment.

In the present multi-factorial MRI study, we investigated simultaneous associations between quantitative measures of medial temporal lobe atrophy and WMH and neuropsychological measures of global cognition, memory, language ability and psychomotor speed in a sample of subjects representing the cognitive continuum of normal cognition, MCI and AD.

\section{Materials and Methods}

\section{Patients}

Elderly referred to the outpatient memory clinic of the Leiden University Medical Center participated in this study. All were evaluated for memory complaints using a standardized dementia screening that included a detailed medical history, a general inter- nal and neurologic exam, laboratory tests, neuropsychological testing and MRI. Diagnoses were made in a multidisciplinary consensus meeting. Forty-one patients received a diagnosis of mild to moderate probable AD [1]. MCI was observed in 20 patients [2, 23]. In addition, 28 elderly controls without memory complaints were recruited through an advertisement in a local newspaper. Controls were evaluated in the same way as patients. Patients and controls were excluded if they had neurologic or psychiatric comorbidity, and if they had abnormalities on MRI other than WMH or an incidental small lacunar lesion ( $\leq 5 \mathrm{~mm}$ in diameter). The study was approved by the local Medical Ethical Committee. Written informed consent was obtained from all subjects or from a close relative if a patient was demented.

\section{Neuropsychological Assessment}

Cognitive functions were assessed using a standardized neuropsychological test battery. From this test battery, we selected four neuropsychological tests that reflect the most important cognitive domains. All are quantitative in nature and widely used in studies assessing dementia. The Cambridge Cognitive Examination (CAMCOG) was used to assess global cognitive functioning [24, 25]. Memory function was evaluated using the Wechsler Memory Scale (WMS) [26]. The Boston naming task was employed to yield a measure of language ability [27]. Psychomotor speed was measured using the Trail Making Test, which consists of a simple (Trails A) and a complex (Trails B) condition [28].

\section{MRI Data}

MRI was performed on a 1.5-tesla MR system (Philips Medical Systems, Best, The Netherlands). $\mathrm{T}_{1}$-weighted 3D gradient echo ( 120 coronal slices; slice thickness $=3 \mathrm{~mm}$; overlap $=1.5 \mathrm{~mm}$; TR/ TE 30/4.6 ms; flip angle $=30^{\circ}$; field of view $=220 \mathrm{~mm}$; matrix 256 $\times 256$ ), dual fast spin-echo (48 axial slices; slice thickness $=3 \mathrm{~mm}$; no gap; TR/TE 3,000/27/120 ms; flip angle $=90^{\circ}$; field of view $=$ $220 \mathrm{~mm}$; matrix $256 \times 256)$ and fast fluid attenuated inversion recovery (FLAIR) sequences were obtained in all patients. The line through the inferior border of the genu and splenium of the corpus callosum defined the direction of scanning for the dual echo and FLAIR images. The direction of scanning for the $\mathrm{T}_{1}$-weighted sequence was perpendicular to this line.

In the diagnostic work-up, MRI was used to rule out other causes of cognitive impairment. For research purposes only, the following quantitative MRI measures were obtained. The $\mathrm{T}_{1}$-weighted images were used to obtain intracranial, whole brain and medial temporal lobe volume, whereas the dual echo sequences were used to obtain WMH volume. All measurements were performed by a single rater who was blind to subject identity and diagnosis.

Manual segmentation of the medial temporal lobe (including hippocampus and parahippocampal gyrus) was performed using the interactive software package DISPLAY (Brain Imaging Center of the Montreal Neurological Institute) [29]. Boundaries were adapted from Watson et al. [30, 31]. Briefly, segmentation of the hippocampus started when the mammillary bodies became visible and ended when the fornix formed a continuous tract. The parahippocampal gyrus started three slices anterior to the hippocampus. The posterior commissure marked its posterior border. Left and right hippocampus and parahippocampal gyrus were summed to obtain the total medial temporal lobe volume.

Semi-automated segmentation of intracranial and whole brain volume and quantification of WMH volume was performed using 
Table 1. Sociodemographic characteristics, neuropsychological test results and MRI measures by diagnostic group

\begin{tabular}{lcccc}
\hline & Control & MCI & AD & $\mathrm{p}^{\mathrm{a}}$ \\
\hline Males/females & $12 / 16$ & $6 / 14$ & $15 / 26$ & $0.66^{\mathrm{b}}$ \\
Age, years & $75(7)$ & $73(8)$ & $74(7)$ & 0.81 \\
Education, years & $9(3)$ & $10(3)$ & $10(3)$ & 0.37 \\
CAMCOG & $96(4)$ & $83(6)$ & $65(10)$ & 0.00 \\
WMS & $127(12)$ & $92(7)$ & $80(11)$ & 0.00 \\
Boston Naming Task & $25(3)$ & $21(4)$ & $17(5)$ & 0.00 \\
Trails A & $34(11)$ & $54(18)$ & $117(82)$ & 0.00 \\
Trails B & $83(33)$ & $195(132)$ & $403(125)$ & 0.00 \\
Intracranial volume, $\mathrm{cm}^{3}$ & $1,406(132)$ & $1,413(131)$ & $1,401(116)$ & 0.94 \\
Whole brain volume, $\mathrm{cm}^{3}$ & $1,064(125)$ & $1,056(127)$ & $1,021(118)$ & 0.32 \\
Medial temporal lobe, $\mathrm{cm}^{3}$ & $8.7(1)$ & $7.5(2)$ & $6.9(1)$ & 0.00 \\
WMH, cm & & $7.0(9)$ & $10.0(13)$ & $0.13^{\mathrm{c}}$ \\
\hline
\end{tabular}

Data are presented as mean (SD).

${ }^{a}$ Calculated with ANOVA unless stated otherwise.

${ }^{\mathrm{b}} \chi^{2}$ test.

c Kruskal-Wallis test. in-house developed software [9]. Segmentations of WMH were generated automatically. Subsequently, the automatically detected lesions were edited manually to correct for incidental inclusion of cerebrospinal fluid, gray matter and Virchow-Robin spaces. WMH were defined as hyperintense lesions on both proton density and $\mathrm{T}_{2}$-weighted images. FLAIR hard copies were used as a reference to minimize misclassification. Infratentorial lesions were excluded. To assess the reliability of the automated lesion detection of the software, 9 patients underwent scan-rescan with and without repositioning procedures. Intraclass correlation coefficients (ICC) were $>0.75$. In addition, ten randomly selected brains were segmented twice to assess intrarater reliabilities of the medial temporal lobe and $\mathrm{WMH}$ volume (ICC for medial temporal lobe volume $=0.91$ and for WMH $>0.99$ ) and interrater reliability of intracranial and whole brain volume (ICC intracranial volume $=0.97$, whole brain volume $=1.0$ ).

\section{Statistical Analysis}

SPSS for Windows (release 10.0; SPSS, Chicago, Ill., USA) was used for data analysis. Group differences were analyzed using analysis of variance (ANOVA) with post-hoc Bonferroni tests, $\chi^{2}$ tests and Kruskal-Wallis tests when appropriate. Univariate associations were assessed using Pearson's correlation analysis. Multiple linear regression analyses were performed to examine independent associations between MRI measures and cognitive impairment. Neuropsychological test results were used as dependent variables. Medial temporal lobe volume and WMH were the predictors. In addition, age, sex and whole brain volume were entered as covariates. The level of statistical significance was set at $\mathrm{p}<0.05$.

\section{Results}

The sociodemographic characteristics, neuropsychological test results and brain measures of the subjects are presented in table 1. Diagnostic groups did not differ with respect to sex, age or education. There were significant differences between groups for all neuropsychological tests. Patients with AD scored significantly lower than patients with MCI and controls on all neuropsychological tests ( $\mathrm{AD}<$ controls: all $\mathrm{p}<0.001 ; \mathrm{AD}<\mathrm{MCI}$ : $\mathrm{p}<0.007$ ). Patients with MCI had intermediate scores between AD patients and controls. MCI patients had significantly lower scores than controls on all tests $(p<0.005)$ with the exception of Trails A $(p=0.68)$. Medial temporal lobe volumes were significantly different between groups. Patients with $\mathrm{AD}$ and $\mathrm{MCI}$ had significantly smaller medial temporal lobes than controls ( $\mathrm{MCI}<$ controls: $\mathrm{p}=0.015$; $\mathrm{AD}<$ controls: $\mathrm{p}<0.001)$. There were no significant differences between groups in intracranial volume, whole brain volume or WMH.

Across groups, all neuropsychological tests were significantly correlated, and there was a significant correlation between medial temporal lobe volume and WMH (table 2). In the univariate analysis, all neuropsychological tests were correlated with medial temporal lobe volume and WMH, except for the WMS, which was not correlated with WMH (fig. 1).

Multiple linear regression analyses were performed to investigate the independent contribution of medial temporal lobe volume and $\mathrm{WMH}$ to impairment in several 

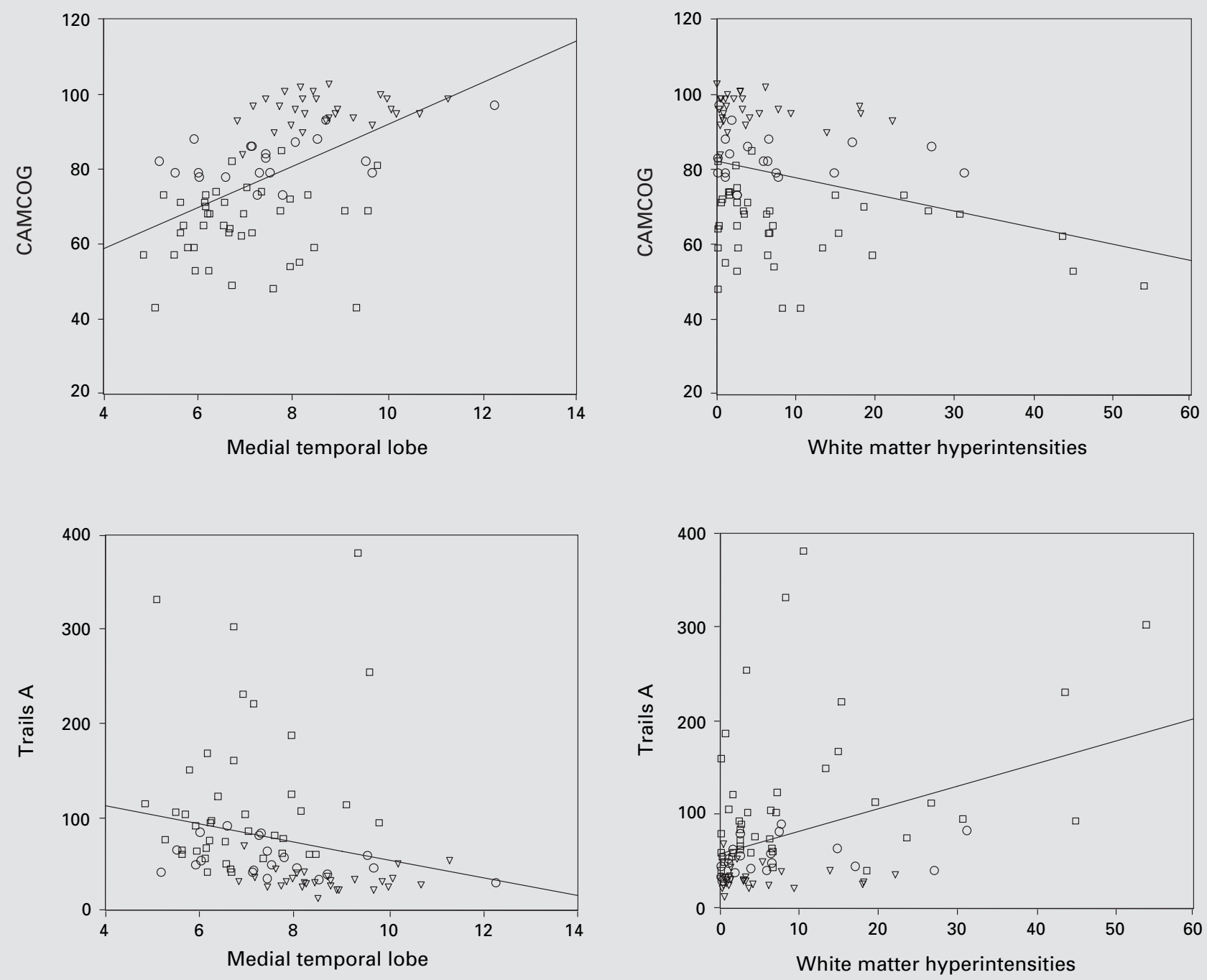

Fig. 1. Scatterplots of CAMCOG and Trails A by medial temporal lobe volume and WMH. $\square=\mathrm{AD} ; \bigcirc=\mathrm{MCI} ; \nabla=$ control.

Table 2. Correlations between neuropsychological measures and MRI measures $(n=89)$

\begin{tabular}{lccccccc}
\hline & CAMCOG & WMS & Boston & Trails A & Trails B & MTL & WMH \\
\hline CAMCOG & 1.00 & & & & & & \\
WMS & $0.88^{* *}$ & 1.00 & & & & & \\
Boston & $0.76^{* *}$ & $0.66^{* *}$ & 1.00 & & & & \\
Trails A & $-0.66^{* *}$ & $-0.56^{* *}$ & $-0.47^{* *}$ & 1.00 & & & \\
Trails B & $-0.79^{* *}$ & $-0.76^{* *}$ & $-0.57^{* *}$ & $0.64^{* *}$ & 1.00 & & \\
MTL & $0.53^{* *}$ & $0.45^{* *}$ & $0.51^{* *}$ & $-0.21^{*}$ & $-0.39^{* *}$ & 1.00 & \\
WMH & $-0.30^{* *}$ & -0.21 & $-0.35^{* *}$ & $0.38^{* *}$ & $0.22^{*}$ & $-0.27^{*}$ & 1.00 \\
\hline
\end{tabular}

Values are Pearson correlation coefficients. $* \mathrm{p}<0.05 ; * * \mathrm{p}<0.01$ 
cognitive domains (table 3 ). In addition to medial temporal lobe volume and $\mathrm{WMH}$, age, sex and whole brain volume were entered as covariates in all analyses. Interaction terms for medial temporal lobe volume and WMH were not significant, and were therefore left out of the final analyses. Both medial temporal lobe volume and WMH were independently associated with scores on the CAMCOG and the Boston naming task. Medial temporal lobe volume was selectively associated with the WMS and Trails B, whereas WMH volume was selectively associated with Trails A. These results did not change essentially when MRI measures were normalized to intracranial volume (data not shown).

\section{Discussion}

The main finding of the present study is that medial temporal lobe atrophy and WMH independently and specifically affect cognitive decline at old age. Memory function, as measured using the WMS, and performance on Trails B was preferably associated with medial temporal lobe volume, and psychomotor slowness as measured using Trails A with WMH. Both MRI measures contributed to the CAMCOG and Boston naming task, measures of global cognitive impairment and language disability, respectively.

We recently demonstrated that the combination of medial temporal lobe atrophy and WMH is associated with the presence of dementia [9]. In the present study, we extend these findings, as both MRI measures were shown to independently contribute to the severity of dementia as measured using neuropsychological tests. In contrast with our former study, our current results do not suggest an interaction between medial temporal lobe atrophy and WMH. However, we do not think the two studies are contradictory as they both clearly indicate that WMH and medial temporal lobe atrophy are important contributors to the presence and severity of dementia.

There have been a number of studies addressing the associations between single MRI measures and impairment in cognitive domains. Medial temporal lobe atrophy has repeatedly been shown to be associated with cognitive decline, especially memory impairment [13-15]. However, there is inconsistency in the literature concerning the cognitive correlates of WMH [18-21]. Psychomotor speed has been mentioned in this respect, but other studies did not find significant associations between $\mathrm{WMH}$ and cognitive impairment [17]. In contrast with the above-mentioned mono-factorial studies, the number
Table 3. Associations between medial temporal lobe volume (MTL) and $\mathrm{WMH}$ and neuropsychological test results $(\mathrm{n}=89)$

\begin{tabular}{lccl}
\hline & $\mathrm{R}^{2}$ & $\beta(\mathrm{se})_{\mathrm{MTL}}$ & $\beta(\mathrm{se})_{\mathrm{WMH}}$ \\
\hline CAMCOG & 0.37 & $8.5(1.6)^{* *}$ & $-3.9(1.5)^{*}$ \\
WMS & 0.32 & $12.3(2.4)^{* *}$ & $-4.5(2.3)$ \\
Boston Naming Task & 0.33 & $2.9(0.6)^{* *}$ & $-1.5(0.6)^{* *}$ \\
Trails A & 0.20 & $-10.5(7.6)$ & $28.2(7.3)^{* *}$ \\
Trails B & 0.21 & $-65.7(19.8)^{* *}$ & $33.0(19.0)$
\end{tabular}

Linear regression analyses with cognitive tests as dependent variables were performed. MTL, WMH, age, sex and whole brain were entered as independent variables. Regression coefficients were standardized to enable direct comparison of their effects on cognitive functions. The regression coefficients represent decrease in cognitive test result with decrease in one standard deviation of MTL or $\mathrm{WMH} . * \mathrm{p}<0.05 ; * * \mathrm{p}<0.01$.

of studies simultaneously investigating medial temporal lobe atrophy and WMH in relation to impairment in specific cognitive functions is limited [32].

There has been one earlier report examining the cognitive correlates of both hippocampal atrophy and WMH in a sample of subjects covering a broad range of cognitive functioning [32]. In that study, hippocampal volume was a strong independent predictor of several cognitive functions, whereas WMH were weakly associated only with fluency. In contrast with these findings, we observed independent associations of both medial temporal lobe atrophy and $\mathrm{WMH}$ with multiple cognitive functions. Although we consistently observed associations between $\mathrm{WMH}$ and cognitive functioning, the associations were of moderate strength. The contrasting results may be explained by our highly reliable method of WMH quantification, that enabled us to reveal these subtle associations. Furthermore, the differences in study sample may be responsible for the contrasting findings. In both studies, subjects with a broad variability of cognitive impairment were included. However, the group of nondemented subjects was overrepresented (81\%) in the earlier study, whereas almost half of the participants in the present study consisted of demented patients. It may be speculated that hippocampal atrophy is a prerequisite to develop the first signs of cognitive decline. Once there is $\mathrm{MCI}$, the presence of $\mathrm{WMH}$ increases the burden of pathology, thus further worsening cognitive function. This notion is concordant with the brain reserve theory, according to which brain injury may add up until a certain threshold is reached and clinical dementia occurs $[6,33]$. 
It also agrees with the study by Wu et al. [34], who report that WMH are strongly associated with the presence of dementia.

Strengths of the present study include the broad range of cognitive functioning that was covered, enabling us to appreciate the full spectrum of structural-functional relationships. Quantitative measures of medial temporal lobe atrophy and WMH were used, to assess their independent contributions to cognitive impairment in a sensitive and reliable way. The neuropsychological tests used for this study are widely used to assess cognitive functions in memory clinics. Although each test was chosen to measure functioning in a specific cognitive domain, there is in fact substantial overlap between cognitive domains. Therefore, reduced performance on a specific test may be caused by impairment in several cognitive domains. For example, Trails B is essentially a test of complex psychomotor speed and executive functioning. However, due to the complex test instructions, it also places a considerable demand on the memory. Moreover, we interpreted the association between medial temporal lobe volume and Trails B as being dependent on memory function, as in our study sample, Trails B seemed to be more strongly associated with the memory test than with the test for simple psychomotor speed. Among the limitations are the small patient numbers, and results need to be replicated in larger samples. Furthermore, we assessed the effect of total WMH on cognitive functions. It is conceivable that regional measures of $\mathrm{WMH}$, like periventricular and subcortical WMH, would have been more specific for specific aspects of cognition.

In conclusion, we demonstrated that MRI measures suggestive of Alzheimer-type and microvascular pathology independently affect cognitive impairment at old age. Neuropsychological measures of global cognitive function and language ability were associated with both medial temporal lobe atrophy and WMH. Memory impairment was preferentially associated with medial temporal lobe atrophy, whereas performance on a test of psychomotor slowness mainly depended on WMH. These results support the view that Alzheimer-type and microvascular brain pathology significantly contribute to cognitive decline at old age. Moreover, these data provide evidence that vascular pathology and Alzheimer-type pathology each have specific cognitive correlates in the elderly.

\section{Acknowledgements}

We thank Dominique van den Heuvel for WMH training. Rijckert van der Flier is gratefully acknowledged for general support and critical comments. We thank Drs. R. van den Boom, A. Spilt, M.C. Kruit and S.C.A. Steens for performing the MRI scans of control subjects. We thank the Internationale Stichting Alzheimer Onderzoek (ISAO) for financial support.

\section{References}

1 McKhann G, Drachman D, Folstein M, Katzman R, Price D, Stadlan EM: Clinical diagnosis of Alzheimer's disease: Report of the NINCDS-ADRDA Work Group under the auspices of Department of Health and Human Services Task Force on Alzheimer's Disease. Neurology 1984;347:939-944.

-2 Petersen RC, Smith GE, Ivnik RJ, Tangalos EG, Schaid DJ, Thibodeau SN, et al: Apolipoprotein $\mathrm{E}$ status as a predictor of the development of Alzheimer's disease in memory-impaired individuals [published erratum appears in JAMA 1995 Aug 16;2747:538]. JAMA 1995;273:1274-1278.

-3 Petersen RC, Stevens JC, Ganguli M, Tangalos EG, Cummings JL, DeKosky ST: Practice parameter: Early detection of dementia: Mild cognitive impairment (an evidence-based review): Report of the Quality Standards Subcommittee of the American Academy of Neurology. Neurology 2001;569:1133-1142.
4 Neuropathology Group of the Medical Research Council Cognitive Function and Ageing Study (MRC CFAS): Pathological correlates of late-onset dementia in a multicentre, community-based population in England and Wales Lancet 2001;357:169-175.

-5 Snowdon DA, Greiner LH, Mortimer JA, Riley KP, Greiner PA, Markesbery WR: Brain infarction and the clinical expression of Alzheimer disease. The Nun Study. JAMA 1997; 277:813-817.

-6 Esiri MM, Nagy Z, Smith MZ, Barnetson L, Smith AD: Cerebrovascular disease and threshold for dementia in the early stages of Alzheimer's disease. Lancet 1999;354:919-920.

7 Gosche KM, Mortimer JA, Smith CD, Markesbery WR, Snowdon DA: Hippocampal volume as an index of Alzheimer neuropathology: Findings from the Nun Study. Neurology 2002;58:1476-1482.

-8 Pantoni L, Garcia JH: Pathogenesis of leukoaraiosis: A review. Stroke 1997;28:652-659.
-9 van der Flier WM, Middelkoop HA, Weverling-Rijnsburger AW, Admiraal-Behloul F, Spilt A, Bollen EL, et al: Interaction of medial temporal lobe atrophy and white matter hyperintensities in AD. Neurology 2004;62:18621864.

10 de Leeuw FE, Barkhof F, Scheltens P: White matter lesions and hippocampal atrophy in Alzheimer's disease. Neurology 2004;62:310_ 312 .

11 Jack CRJ, Petersen RC, Xu YC, Waring SC, O'Brien PC, Tangalos EG, et al: Medial temporal atrophy on MRI in normal aging and very mild Alzheimer's disease. Neurology 1997;49:786-794.

12 Krasuski JS, Alexander GE, Horwitz B, Daly EM, Murphy DG, Rapoport SI, et al: Volumes of medial temporal lobe structures in patients with Alzheimer's disease and mild cognitive impairment (and in healthy controls). Biol Psychiatry 1998;43:60-68. 
$\checkmark 13$ Deweer B, Lehéricy S, Pillon B, Baulac M, Chiras $\mathrm{J}$, Marsault $\mathrm{C}$, et al: Memory disorders in probable Alzheimer's disease: The role of hippocampal atrophy as shown with MRI. J Neurol Neurosurg Psychiatry 1995;58:590-597.

-14 Fama R, Sullivan EV, Shear PK, Marsh L, Yesavage JA, Tinklenberg JR, et al: Selective cortical and hippocampal volume correlates of Mattis Dementia Rating Scale in Alzheimer disease. Arch Neurol 1997;54:719-728.

$\checkmark 15$ Petersen RC, Jack CRJ, Xu YC, Waring SC, O'Brien PC, Smith GE, et al: Memory and MRI-based hippocampal volumes in aging and AD. Neurology 2000;54:581-587.

16 Squire LR, Zola-Morgan S: The medial temporal lobe memory system. Science 1991;253: 1380-1386.

$>17$ de Groot JC, de Leeuw FE, Oudkerk M, van Gijn J, Hofman A, Jolles, J et al: Cerebral white matter lesions and cognitive function: The Rotterdam Scan Study. Ann Neurol 2000;47: 145-151.

- 18 O'Brien JT, Wiseman R, Burton EJ, Barber B, Wesnes K, Saxby B, et al: Cognitive associations of subcortical white matter lesions in older people. Ann NY Acad Sci 2002; 977:436444.

19 Hirono N, Kitagaki H, Kazui H, Hashimoto M, Mori E: Impact of white matter changes on clinical manifestation of Alzheimer's disease: A quantitative study. Stroke 2000;31:21822188 .
20 Barber R, Scheltens P, Gholkar A, Ballard C, McKeith I, Ince P, et al: White matter lesions on magnetic resonance imaging in dementia with Lewy bodies, Alzheimer's disease, vascular dementia, and normal aging. J Neurol Neurosurg Psychiatry 1999;67:66-72.

21 Stout JC, Jernigan TL, Archibald SL, Salmon DP: Association of dementia severity with cortical gray matter and abnormal white matter volumes in dementia of the Alzheimer type. Arch Neurol 1996;53:742-749.

22 Scheltens P, Barkhof F, Valk J, Algra PR, van der Hoop RG, Nauta J, et al: White matter lesions on magnetic resonance imaging in clinically diagnosed Alzheimer's disease. Evidence for heterogeneity. Brain 1992;115:735-748.

23 Petersen RC, Smith GE, Waring SC, Ivnik RJ Tangalos EG, Kokmen E: Mild cognitive impairment: Clinical characterization and outcome. Arch Neurol 1999;56:303-308.

24 Roth M, Tym E, Mountjoy CQ, Huppert FA, Hendrie H, Verma S, et al: CAMDEX. A standardised instrument for the diagnosis of mental disorder in the elderly with special reference to the early detection of dementia. Br J Psychiatry 1986;149:698-709.

25 Derix M, Hofstede A, Teunisse S, et al: CAMDEX-N: De Nederlandse Versie van de Cambridge Examination of Mental Disorders of the Elderly. Lisse, Zwets \& Zeitlinger, 1992.

26 Wechsler DA: A standardized memory scale for clinical use. J Psychol 1945;19:87-95.

27 Kaplan EF, Goodglass H, Weintraub S: The Boston Naming Test. Boston, Kaplan and Goodglass, 1978.
28 Reitan RM: Validity of the Trail Making Test as an indicator of organic brain damage. Percept Mot Skills 1958;8:271-276.

29 Pruessner JC, Kohler S, Crane J, Pruessner M, Lord C, Byrne A, et al: Volumetry of temporopolar, perirhinal, entorhinal and parahippocampal cortex from high-resolution MR images: Considering the variability of the collateral sulcus. Cereb Cortex 2002; 12:13421353.

30 Staal WG, Hulshoff Pol HE, Schnack H, Hoogendoorn MLC, Jellema K, Kahn RS: Structural brain abnormalities in patients with schizophrenia and their healthy siblings. Am J Psychiatry 2000;157:416-421.

>31 Watson C, Andermann F, Gloor P, Jones-Gotman M, Peters T, Evans A, et al: Anatomic basis of amygdaloid and hippocampal volume measurement by magnetic resonance imaging. Neurology 1992;42:1743-1750.

32 Mungas D, Jagust WJ, Reed BR, Kramer JH, Weiner MW, Schuff N, et al: MRI predictors of cognition in subcortical ischemic vascular disease and Alzheimer's disease. Neurology 2001;57:2229-2235.

-33 Satz P: Brain reserve capacity on symptom onset after brain injury: A formulation and review of evidence for threshold theory. Neuropsychology 1993;7:273-295.

>34 Wu CC, Mungas D, Petkov CI, Eberling JL, Zrelak PA, Buonocore MH, et al: Brain structure and cognition in a community sample of elderly Latinos. Neurology 2002;59:383-391. 